\title{
2D fuzzy anti-de Sitter space from matrix models
}

\section{Danijel Jurman $^{a}$ and Harold Steinacker ${ }^{b}$}

${ }^{a}$ Theoretical Physics Division, Rudjer Boskovic Institute P.O. Box 180, 10002 Zagreb, Croatia

${ }^{b}$ Faculty of Physics, University of Vienna

Boltzmanngasse 5, A-1090 Vienna, Austria

E-mail: Danijel.Jurman@irb.hr, harold.steinacker@univie.ac.at

Abstract: We study the fuzzy hyperboloids $A d S^{2}$ and $d S^{2}$ as brane solutions in matrix models. The unitary representations of $\mathrm{SO}(2,1)$ required for quantum field theory are identified, and explicit formulae for their realization in terms of fuzzy wavefunctions are given. In a second part, we study the $(A) d S^{2}$ brane geometry and its dynamics, as governed by a suitable matrix model. In particular, we show that trace of the energy-momentum tensor of matter induces transversal perturbations of the brane and of the Ricci scalar. This leads to a linearized form of Henneaux-Teitelboim-type gravity, illustrating the mechanism of emergent gravity in matrix models.

KeYwords: Non-Commutative Geometry, M(atrix) Theories, 2D Gravity

ARXIV EPRINT: 1309.1598 


\section{Contents}

1 Introduction 1

2 Classical two-dimensional hyperboloid $\quad 2$

2.1 Geometry and isometry group 2

2.2 Functions and Poisson bracket 4

$\begin{array}{lll}3 & \text { Fuzzy hyperboloid } & 6\end{array}$

$\begin{array}{lll}3.1 & \text { Fuzzy wavefunctions } & 7\end{array}$

$\begin{array}{lll}3.2 & \text { Semi-classical limit } & 10\end{array}$

4 Dynamical matrix models $\quad 12$

5 Fluctuating $A d S^{2}$ and gravity $\quad \mathbf{1 5}$

$\begin{array}{lll}5.1 \text { Curvature perturbations and gravity } & 18\end{array}$

$\begin{array}{ll}5.2 \text { Induced metric curvature } & 19\end{array}$

5.3 Gauge theory point of view 20

$\begin{array}{llr}6 & \text { Conclusion } & 21\end{array}$

A Appendix: derivation of the linearized gravity equations 22

\section{Introduction}

There has been a great amount of work on noncommutative field theory on the fuzzy sphere and similar compact quantum spaces. Part of their appeal stems from the fact that the space of functions on these spaces has a simple group-theoretical structure and is finitedimensional, reflecting their finite symplectic volume. This leads to mathematically wellcontrolled toy models for noncommutative field theory and geometry, see e.g. [1-10] and references therein. However, most of the work so far has been for spaces with Euclidean signature, and it would be desirable to know more about fuzzy spaces with Minkowski signature.

In this paper, we study in detail 2-dimensional fuzzy de Sitter space $d S^{2}$ and Antide Sitter space $A d S^{2}$, which are quantized homogeneous spaces with Minkowski signature and non-vanishing curvature. Fuzzy $(A) d S^{2}$ has been studied previously in [11-13]; see also $[14,15]$ for related work on fuzzy noncompact coadjoint orbits, and [16] for a different approach to fuzzyness on $d S^{4}$. In the first part of this paper, we elaborate the space of functions on these fuzzy hyperboloids, and provide explicit formulae for the squareintegrable wavefunctions corresponding to unitary irreducible representations of $\mathrm{SO}(2,1)$. For the discrete series representations we recover previous results obtained in [11], and 
for the principal continuous representations our results are new. This provides the basic constituents for quantum field theory ${ }^{1}$ on fuzzy $A d S^{2}$ and $d S^{2}$. In particular, this also allows to establish the required quantization map for the fuzzy geometry.

In a second part, we consider a matrix model which describes dynamical fuzzy $A d S^{2}$ and $d S^{2}$ spaces as brane solutions. As discussed in [19, 20], this leads to a dynamical effective geometry on the branes, determined by a combination of the embedding geometry of the brane and its Poisson structure. The present 2-dimensional example provides an interesting toy model for emergent gravity, with a non-trivial curvature background. We study the perturbations around the $A d S^{2}$ solutions, and their dynamics in the presence of matter. This is interesting because the extrinsic curvature of the brane leads to a coupling of the linearized matrix perturbations to the energy-momentum tensor, ${ }^{2}$ as pointed out in [21-23]. More precisely, the transversal perturbations of the brane couple to the trace of the energy-momentum tensor of matter, due to the extrinsic curvature. It turns out that the perturbations of the effective metric are governed by a linearized Henneaux-Teitelboimtype gravity $[24,25]$, relating the trace of the energy-momentum tensor to the Ricci scalar. This is remarkable, because it results directly from the underlying matrix model action, without adding any gravity action. It provides a simple example for the mechanism of emergent gravity in Yang-Mills matrix models. However, this result is restricted to the linearized regime.

In 4 and higher dimensions, the dynamics of the effective geometry is complicated due to a mixing between tangential and transversal brane perturbations [21, 22], which prohibits a full understanding at present. A similar mixing is observed here, but we are able to disentangle the coupled wave equations, and thereby essentially solve the perturbative dynamics. Therefore the present 2-dimensional case should serve as a useful step towards understanding the more complicated higher-dimensional case.

\section{Classical two-dimensional hyperboloid}

\subsection{Geometry and isometry group}

There are three types of two-dimensional non-compact spaces with constant curvature, given by the Anti-de Sitter space $A d S^{2}$, de Sitter space $d S^{2}$ and the hyperbolic or Lobachevsky plane $H^{2}$. In this paper we discuss $A d S^{2}$ and $d S^{2}$, which can be naturally realized as the one-sheeted hyperboloid embedded in $\mathbb{R}^{3}$ through

$$
x^{a} x^{b} \eta_{a b}=-\left(x^{1}\right)^{2}-\left(x^{2}\right)^{2}+\left(x^{3}\right)^{2}=-R^{2} .
$$

In terms of conformal coordinates $-\pi / 2<\sigma<\pi / 2$ and $\pi<\tau \leq \pi$, the embedding of classical Anti-de Sitter space $A d S^{2}$ is given by

$$
x^{1}=R \frac{\cos \tau}{\cos \sigma}, x^{2}=R \frac{\sin \tau}{\cos \sigma}, x^{3}=R \tan \sigma .
$$

\footnotetext{
${ }^{1}$ For a discussion in the undeformed case see e.g. $[17,18]$ and references therein.

${ }^{2}$ Rather than just its derivative, as on trivially embedded branes.
} 
The induced metric is pseudo-Riemannian

$$
\begin{array}{lll}
g_{\mu \nu}=\eta_{a b} \partial_{\mu} x^{a} \partial_{\nu} x^{b}, & \eta_{a b}=\operatorname{diag}(-1,-1,1), & \mu, \nu=\sigma, \tau, \\
g_{\tau \tau}=\frac{R^{2}}{\cos ^{2} \sigma}, & g_{\sigma \sigma}=-\frac{R^{2}}{\cos ^{2} \sigma}, & g_{\sigma \tau}=0,
\end{array}
$$

with closed time-like circles ${ }^{3}$ around $x^{3}=$ const . De Sitter space $d S^{2}$ is obtained from $A d S^{2}$ by switching the roles of the time and space, thus changing the overall sign in the metric. The circles $x^{3}=$ const are then space-like, and there are no closed time-like curves.

Both $A d S^{2}$ and $d S^{2}$ admit the group $\mathrm{SO}(2,1)$ or its cover $\mathrm{SU}(1,1)$ as isometries, generated by vector fields $K^{a}, a=1,2,3$

$$
K^{1}=-i \cos \tau \sin \sigma \partial_{\tau}-i \sin \tau \cos \sigma \partial_{\sigma}, K^{2}=-i \sin \tau \sin \sigma \partial_{\tau}+i \cos \tau \cos \sigma \partial_{\sigma}, K^{3}=-i \partial_{\tau},
$$

which close $s u(1,1)$ Lie algebra with respect to commutators

$$
\left[K^{a}, K^{b}\right]=i f_{c}^{a b} K^{c}
$$

or explicitly

$$
\left[K^{1}, K^{2}\right]=-i K^{3},\left[K^{2}, K^{3}\right]=i K^{1},\left[K^{3}, K^{1}\right]=i K^{2} .
$$

The Casimir operator of $s u(1,1)$ Lie algebra is defined as

$$
C=-\left(K^{1}\right)^{2}-\left(K^{2}\right)^{2}+\left(K^{3}\right)^{2} .
$$

As usual, it is convenient to introduce the ladder operators

$$
K^{ \pm}=K^{1} \pm i K^{2}
$$

which satisfy the commutation relations

$$
\left[K^{3}, K^{ \pm}\right]= \pm K^{ \pm},\left[K^{+}, K^{-}\right]=-2 K^{3} .
$$

Then unitary irreducible representations of $\mathrm{SO}(2,1)$ are spanned by a basis $|j, m\rangle$ of weight states, where $j$ is related to the eigenvalue of the Casimir $C$, and $m$ is the eigenvalue of $K^{3}$ and the action of $K^{ \pm}$on $|j, m\rangle$ produces a state with weight $m \pm 1$ :

$$
K^{3} K^{ \pm}|j, m\rangle=(m \pm 1) K^{ \pm}|j, m\rangle \sim|j, m \pm 1\rangle .
$$

A chain of states obtained by the successive action of $\mathrm{K}^{-}$operator terminates if there exist state such that

$$
K^{-}\left|j, m_{0}\right\rangle=0
$$

Denoting this lowest weight by $j=m_{0}$, it follows that

$$
0=K^{+} K^{-}|j, j\rangle=\left(-C+K^{3}\left(K^{3}-1\right)\right)|j, j\rangle \Rightarrow C=j(j-1)
$$

\footnotetext{
${ }^{3}$ They can be avoided by passing to the universal cover of $A d S^{2}$.
} 
Therefore the chain of states which span this irreducible lowest weight representation is determined by the state $|j, j\rangle$ of lowest weight, via

$$
|j, j+m\rangle \sim K^{+m}|j, j\rangle .
$$

By analogy, the highest weight representation are obtained by interchanging roles of $\mathrm{K}^{+}$ and $K^{-}$operators. If no lowest or highest weight state exists, then the normalisability condition implies $C<0$, and the states belong to the unitary irreducible continuous representations.

In general, the resulting structure of irreducible representations is as follows:

$$
\begin{aligned}
K^{3}|j, m\rangle & =m|j, m\rangle, \\
K^{+}|j, m\rangle & =a_{m+1}|j, m+1\rangle, \\
K^{-}|j, m\rangle & =a_{m}|j, m-1\rangle,
\end{aligned}
$$

where

$$
a_{m}=\sqrt{m(m-1)-j(j-1)} .
$$

The finite-dimensional irreducible representations of $\mathrm{SU}(1,1)$ are obtained for $j \in-\mathbb{N} / 2$. They are not unitary, and correspond to the spin $|j|$ representations $V_{|j|}$ of $\mathrm{SU}(2)$ with $C=-|j|(|j|+1)$. All unitary irreducible representations are infinite-dimensional, and fall into one of the following classes ${ }^{4}[26]$ :

- The discrete series of the highest and the lowest weight representations

$$
\begin{array}{lll}
D_{j}^{+}, & j \in \mathbb{N}_{>0}: & \mathcal{H}_{j}=\{|j, m\rangle ; m=j, j+1, \cdots ; m \in \mathbb{N}\}, \\
D_{j}^{-}, & j \in \mathbb{N}_{>0}: & \mathcal{H}_{j}=\{|j, m\rangle ; m=-j,-j-1, \cdots ;-m \in \mathbb{N}\},
\end{array}
$$

characterized by $C=j(j-1) \geq 0$.

- The principal continuous series

$$
P_{s}, \quad s \in \mathbb{R}, \quad 0<s<\infty, \quad j=\frac{1}{2}+i s, \quad \mathcal{H}_{j}=\{|j, m\rangle ; m=0, \pm 1, \ldots ; m \in \mathbb{Z}\}
$$

labeled by a real number $s$ and $C=-\left(s^{2}+\frac{1}{4}\right)<-1 / 4$.

- The complementary series

$$
P_{j}^{c}, \quad 1 / 2<j<1, \quad j \in \mathbb{R}, \quad \mathcal{H}_{j}=\{|j, m\rangle ; m=0, \pm 1, \ldots ; m \in \mathbb{Z}\}
$$

with $-1 / 4<C<0$.

\section{$2.2 \quad$ Functions and Poisson bracket}

In order to carry out the quantization of $(A) d S^{2}$, it is useful to organize the space of functions on $(A) d S^{2}$ in terms of irreducible representations of $\mathrm{SU}(1,1)$. This provides at the same time the basis of eigenfunctions of the invariant d'Alembertian $\square_{g}$,

$$
\square_{g}=\frac{1}{\sqrt{|g|}} \partial_{\mu} \sqrt{|g|} g^{\mu \nu} \partial_{\nu}=\frac{\cos ^{2} \sigma}{R^{2}}\left(\partial_{\tau}^{2}-\partial_{\sigma}^{2}\right)=\frac{K^{1^{2}}+K^{2^{2}}-K^{3^{2}}}{R^{2}},
$$

\footnotetext{
${ }^{4}$ We only consider representations with integer weights for simplicity.
} 
which is related to the Casimir operator of $s u(1,1)$. Here $g=\operatorname{det}\left(g_{\mu \nu}\right)$, and $g^{\mu \nu}$ is inverse of the metric. We can thus decompose any function on the hyperboloid into eigenfunctions of $\square_{g}$,

$$
\square_{g} \phi+\alpha \phi=0
$$

and label the solutions by $j$ and $m$ as above. The solutions corresponding to the finitedimensional representations are realized by polynomial functions $\operatorname{Pol}\left(\mathrm{x}^{\mathrm{a}}\right)$; they are of course not normalizable on $(A) d S^{2}$. The square-integrable functions corresponding to unitary irreducible representations are given explicitly in terms of hyper-geometric functions

$$
\phi_{j m}=e^{-i m \tau} \cos ^{j} \sigma\left[a_{2} F_{1}\left(\frac{j+m}{2}, \frac{j-m}{2}, \frac{1}{2} ; \sin ^{2} \sigma\right)+b \sin \sigma_{2} F_{1}\left(\frac{j+m+1}{2}, \frac{j-m+1}{2}, \frac{3}{2} ; \sin ^{2} \sigma\right)\right],
$$

where

$$
C=j(j-1)=R^{2} \alpha
$$

is the Casimir. For $A d S^{2}$, the scalar fields corresponding to positive or negative energy unitary representations belong to the discrete representation $D_{j}^{ \pm}$, with $\alpha>0$. Then the equations for the lowest weight state have a unique solution

$$
\left.\begin{array}{c}
K^{3} \phi_{j j}=j \phi_{j j} \\
K^{-} \phi_{j j}=0
\end{array}\right\} \Rightarrow \phi_{j j} \sim e^{i j \tau} \cos ^{j} \sigma
$$

and the spectrum is non-degenerate. On the other hand the states given by (2.22) with $\alpha<0$ belong to the continuous representations, with two-fold degenerate spectrum. These are the physical scalar fields on de Sitter space $d S^{2}$. Putting these together, we have the following decomposition of functions on the hyperboloid $(A) d S^{2}$

$$
L^{2}\left((A) d S^{2}\right)=\oplus_{J \geq 1} D_{J}^{+} \oplus_{J \geq 1} D_{J}^{-} \oplus 2 \int_{0}^{\infty} d S P_{S}
$$

along with the space of polynomial functions $\operatorname{Pol}\left(\mathrm{x}^{\mathrm{a}}\right)$.

In the following we discuss fuzzy versions of these non-compact spaces, and their associated spaces of functions. As a starting point, we note that the natural $\mathrm{SO}(2,1)$ invariant volume element endows the hyperboloid with a non-degenerate symplectic form

$$
\omega=\frac{R}{\kappa \cos ^{2} \sigma} d \tau \wedge d \sigma
$$

with $d \omega=0$, introducing a scale perameter $\kappa$. Its inverse defines the Poisson bracket of two functions

$$
\{f, g\}=\frac{\kappa \cos ^{2} \sigma}{R}\left(\partial_{\tau} f \partial_{\sigma} g-\partial_{\sigma} f \partial_{\tau} g\right) .
$$

We can now look for a quantization of this Poisson manifold $\mathcal{M}$, cf. [33-36]. This means that the algebra of functions $\mathcal{C}(\mathcal{M})$ should be mapped to a non-commutative (operator) algebra $\mathcal{A}$, such that the commutator is approximated by the Poisson bracket. In the present case, the group-theoretical structure of $(A) d S^{2}$ provides a natural and explicit 
quantization, in analogy to the case of the fuzzy sphere [1]. As a first step, we note that the Poisson brackets of the embedding functions $x^{a}$ satisfy the Lie algebra of $\mathrm{SO}(2,1)$

$$
\left\{x^{a}, x^{b}\right\}=\kappa f_{c}^{a b} x^{c}
$$

where $f_{c}^{a b}$ are structure constants of $\mathrm{SO}(2,1)$. This implies as usual that the corresponding Hamiltonian vector fields satisfy the same Lie algebra, and indeed it is easy to verify that the $\mathrm{SO}(2,1)$ vector fields $(2.5)$ are given by

$$
K^{a}=\frac{i}{\kappa}\left\{x^{a}, .\right\}
$$

\section{$3 \quad$ Fuzzy hyperboloid}

In analogy to the fuzzy sphere [1], we define fuzzy two-dimensional hyperboloid in terms of three hermitian matrices (or operators) $X^{a}$, which are interpreted as quantization of the embedding functions $x^{a}$. In view of (2.28), we impose the following relations

$$
\left[X^{a}, X^{b}\right]=i \kappa f_{c}^{a b} X^{c}
$$

where $f_{c}^{a b}$ are structure constants of the Lie algebra $s u(1,1)$. Therefore the $X^{a}$ are rescaled $s u(1,1)$ generators, and we assume that they act on a certain irreducible unitary representation $\mathcal{H}_{j}$ of the Lie algebra. We can then write the Casimir operator as

$$
X^{a} X^{b} \eta_{a b}=\kappa^{2} j(j-1) .
$$

Since $\mathcal{H}_{j}$ is assumed to be irreducible, the $X^{a}$ generate the full algebra $\mathcal{A}$ of operators on $\mathcal{H}_{j}$

$$
\mathcal{A}:=\operatorname{End}\left(\mathcal{H}_{j}\right) \cong \mathcal{H}_{j} \otimes \mathcal{H}_{j}^{*},
$$

where $\mathcal{H}_{j}^{*}$ is dual representation of $\mathcal{H}_{j}$. This algebra is an infinite-dimensional vector space, which naturally carries an action of $s u(1,1)$ by conjugation with the generators $X^{a}$ :

$$
K^{a} \triangleright \Phi=\frac{1}{\kappa}\left[X^{a}, \Phi\right], \quad \Phi \in \mathcal{A} .
$$

We now specify the representation $\mathcal{H}_{j}$. Since the matrices $X^{a}$ should be interpreted as quantized embedding functions $x^{a}$ of the hyperboloid and comparing the spectrum of $X^{3}$ with the range of $x^{3} \in\langle-\infty, \infty\rangle$, we choose $\mathcal{H}_{j}$ to be a principal continuous representation, ${ }^{5}$ in accord with [11].

We can furthermore define an invariant scalar product

$$
\left(\Phi_{1}, \Phi_{2}\right)=\operatorname{Tr} \Phi_{1}^{\dagger} \Phi_{2}, \quad \Phi_{1}, \Phi_{2} \in \mathcal{A} .
$$

$\mathcal{A}$ contains in particular the polynomials generated by the $X^{a}$, where this trace diverges. However, $\mathcal{A}$ also contains normalizable matrices corresponding to physical scalar fields,

\footnotetext{
${ }^{5}$ The complementary representation is rejected because it does not admit a semi-classical limit for fixed curvature, as explained in section 3.2.
} 
which are of main interest here. Finding such normalizable matrices is equivalent to decomposing $\mathcal{A}=\mathcal{H}_{j} \otimes \mathcal{H}_{j}^{*}$ into irreducible unitary representations of $s u(1,1)$. This problem has been extensively studied in the literature [27-32]. In general, the states $|J M\rangle$ which belong to a particular unitary irreducible representation in $\mathcal{H}_{j_{1}} \otimes \mathcal{H}_{j_{2}}$ are given by

$$
|J M\rangle=\sum_{m_{1}, m_{2}} C_{m_{1} m_{2} M}^{j_{1} j_{2} J}\left|j_{1} m_{1}\right\rangle \otimes\left|j_{2} m_{2}\right\rangle
$$

Here the $C$ 's are the Wigner coefficients, which vanish unless $M=m_{1}+m_{2}$. In the special case of $\mathcal{H}_{j} \otimes \mathcal{H}_{j}^{*}$, we represent the state (3.6) as a matrix $\Phi_{M}^{J}$

$$
\Phi_{M}^{J}=\sum_{m_{1} m_{2}} D_{m_{1} m_{2} M}^{j j J}\left|j m_{1}\right\rangle\left\langle j m_{2}\right|
$$

where the $D$ 's vanish unless $M=m_{1}-m_{2} \in \mathbb{Z}$. Since we chose the principal continuous representation $\mathcal{H}_{j} \cong P_{s}$, one obtains the following decomposition of the space of functions $\mathcal{A}$ into unitary modes $[27-30]$ :

$$
P_{s} \otimes P_{s}^{\prime}=\oplus_{J \geq 1} D_{J}^{+} \oplus_{J \geq 1} D_{J}^{-} \oplus 2 \int_{0}^{\infty} d S P_{S}
$$

along with the space of polynomial functions $\operatorname{Pol}\left(\mathrm{X}^{\mathrm{a}}\right)$. In the next section we will recover this result and obtain the corresponding fuzzy wavefunctions explicitly, which solve the eigenvalue equations

$$
\begin{aligned}
\eta_{a b} K^{a} \triangleright K^{b} \triangleright \Phi_{M}^{J} & =-\frac{1}{\kappa^{2}} \square \Phi_{M}^{J}=\frac{\eta_{a b}}{\kappa^{2}}\left[X^{a},\left[X^{b}, \Phi_{M}^{J}\right]\right]=J(J-1) \Phi_{M}^{J}, \\
K^{3} \triangleright \Phi_{M}^{J} & =\frac{1}{\kappa}\left[X^{3}, \Phi_{M}^{J}\right]=M \Phi_{M}^{J} .
\end{aligned}
$$

\subsection{Fuzzy wavefunctions}

We can determine the fuzzy wavefunctions $\Phi_{M}^{J}$ explicitly, using their definition as irreducible representations of $\mathrm{SO}(2,1)$. As an element of the operator algebra $\mathcal{A}$, the matrix $\Phi_{M}^{J}$ acts on $|j n\rangle \in \mathcal{H}_{j}$ as

$$
\Phi_{M}^{J}|j n\rangle=\sum_{m} D_{m m-M M}^{j \quad j \quad J}|j m\rangle\langle j m-M \mid j n\rangle=D_{n+M n M}^{j \quad j J}|j n+M\rangle
$$

Defining the matrix $D_{M}^{J}\left(K_{3}\right)$ by its action on $|j n\rangle$

$$
D_{M}^{J}\left(K_{3}\right)|j n\rangle=D_{n n-M M}^{j \quad j \quad J}|j n\rangle
$$


and using defining property $\Gamma(x+1)=x \Gamma(x)$ of Gamma function, we can express $\Phi_{M}^{J}$ for integer $M$ using (2.15) as

$$
\begin{aligned}
\Phi_{M}^{J} & =D_{M}^{J}\left(K^{3}\right) \sqrt{\frac{\Gamma\left(K^{3}-M-j+1\right) \Gamma\left(K^{3}-M+j\right)}{\Gamma\left(K^{3}-j+1\right) \Gamma\left(K^{3}+j\right)}} K^{+M}= \\
& =D_{M}^{J}\left(K^{3}\right)\left(\frac{1}{\sqrt{\left(K^{3}-j\right)\left(K^{3}+j-1\right)}} K^{+}\right)^{M}, \quad M>0 \\
\Phi_{M}^{J} & =K^{-M} \sqrt{\frac{\Gamma\left(K^{3}-M-j+1\right) \Gamma\left(K^{3}-M+j\right)}{\Gamma\left(K^{3}-j+1\right) \Gamma\left(K^{3}+j\right)}} D_{M}^{J}\left(K^{3}\right)= \\
& =\left(K^{-} \frac{1}{\sqrt{\left(K^{3}-j\right)\left(K^{3}+j-1\right)}}\right)^{M} D_{M}^{J}\left(K^{3}\right)^{\dagger}, \quad M<0 .
\end{aligned}
$$

To derive the final expressions (3.12) and (3.13) one applies the identity

$$
\sqrt{\frac{\Gamma\left(K^{3}-M-j+1\right) \Gamma\left(K^{3}-M+j\right)}{\Gamma\left(K^{3}-j+1\right) \Gamma\left(K^{3}+j\right)}} K^{+M}=\left(\frac{1}{\sqrt{\left(K^{3}-j\right)\left(K^{3}+j-1\right)}} K^{+}\right)^{M}
$$

which can be verified using

$$
K^{+} F\left(K^{3}\right)=F\left(K^{3}-1\right) K^{+},
$$

which follows from (2.10). We note that the expressions (3.12) and (3.13) are hermitian conjugates of each other. This reflects the fact that $\Phi_{M}^{J}{ }^{\dagger}$ is a solution of (3.9) with eigenvalue $-M$ if $\Phi_{M}^{J}$ is a solution with eigenvalue $M$.

The above considerations apply to any representation. For the discrete series representations $D_{J}^{+}$in (3.8) with $J$ being integer, the basis of states is completely determined by the minimal weight state annihilated by $K^{-}$. Acting with $K^{-}$on $(3.12)$ we obtain

$$
\begin{aligned}
{\left[K^{-}, \Phi_{M}^{J}\right]=} & \sqrt{(M-J)(M+J-1)} \Phi_{M-1}^{J} \\
= & {\left[D_{M}^{J}\left(K^{3}+1\right) \sqrt{\left(K^{3}+j\right)\left(K^{3}-j+1\right)}\right.} \\
& \left.-D_{M}^{J}\left(K^{3}\right) \sqrt{\left(K^{3}-M+j\right)\left(K^{3}-M-j+1\right)}\right] \times \\
& \times\left(\frac{1}{\sqrt{\left(K^{3}-j\right)\left(K^{3}+j-1\right)}} K^{+}\right)^{M-1} .
\end{aligned}
$$

Specializing this to the case $M=J$, we see that the expression in square bracket must vanish

$$
\begin{aligned}
& D_{J}^{J}\left(K^{3}+1\right) \sqrt{\frac{\Gamma\left(K^{3}+j+1\right) \Gamma\left(K^{3}-j+2\right)}{\Gamma\left(K^{3}-J-j+2\right) \Gamma\left(K^{3}-J+j+1\right)}}- \\
& \quad-D_{J}^{J}\left(K^{3}\right) \sqrt{\frac{\Gamma\left(K^{3}+j\right) \Gamma\left(K^{3}-j+1\right)}{\Gamma\left(K^{3}-J+j\right) \Gamma\left(K^{3}-J-j+1\right)}}=0 .
\end{aligned}
$$


Since $K^{3}$ takes only integer values here, we can conclude that

$$
D_{J}^{J}\left(K^{3}\right)=\sqrt{\frac{\Gamma\left(K^{3}-J+j\right) \Gamma\left(K^{3}-J-j+1\right)}{\Gamma\left(K^{3}+j\right) \Gamma\left(K^{3}-j+1\right)}}
$$

(up to normalization). Finally, for the lowest weight state in $D_{J}^{+}$using (3.14) we obtain

$$
\Phi_{J}^{J}=\left(\frac{1}{\left(K^{3}-j\right)\left(K^{3}+j-1\right)} K^{+}\right)^{J}
$$

in agreement with findings of $[11,12]$. The highest weight state in $D_{J}^{-}$is given by hermitian conjugate of (3.19).

For the principal continuous representation $P_{S}$ with $J=1 / 2+i S$ in (3.8), the matrices $D_{M}^{J}$ are solutions of second order difference equation obtained from (3.16) applying $K^{+}$ on it:

$$
\begin{aligned}
& {\left[(M-J)(M+J-1)-\left(K^{3}-j\right)\left(K^{3}+j-1\right)-\left(M-K^{3}-j\right)\left(M-K^{3}+j-1\right)\right] C_{M}^{J}\left(K^{3}\right)} \\
& =\sqrt{\left(K^{3}-j\right)\left(K^{3}+j-1\right)\left(M-K^{3}+j\right)\left(M-K^{3}-j+1\right)} C_{M}^{J}\left(K^{3}-1\right)+ \\
& \quad \sqrt{\left(K^{3}+j\right)\left(K^{3}-j+1\right)\left(M-K^{3}-j\right)\left(M-K^{3}+j-1\right)} C_{M}^{J}\left(K^{3}+1\right) .
\end{aligned}
$$

Here we find it convenient to write $D_{M}^{J}$ as

$$
D_{M}^{J}\left(K^{3}\right)=C_{M}^{J}\left(K^{3}\right) e^{i \pi K^{3}}
$$

where $C_{M}^{J}\left(K_{3}\right)$ is a matrix with elements given by the Wigner coefficients for the principal continuous representations of $s u(1,1)$ in $(3.8)$. This can be seen after noting that this second order difference equation is a special case of equation for the general Wigner coefficients as found in $[31,32]$. Finally, we can express $C_{M}^{J}$ in terms of two independent solutions ${ }^{6}$

$$
\begin{aligned}
C_{M}^{J}\left(K^{3}\right)= & \sqrt{\frac{\Gamma\left(M-K^{3}-j+1\right) \Gamma\left(M-K^{3}+j\right)}{\Gamma\left(K^{3}+j\right) \Gamma\left(K^{3}-j+1\right)}} \times \\
& \times \frac{a G_{M}^{J}\left(K^{3}\right)+b G_{M}^{1-J}\left(K^{3}\right)}{\Gamma\left(M-K^{3}+J-j+1\right) \Gamma\left(M-K^{3}-J-j-2\right)} .
\end{aligned}
$$

where $G_{M}^{J}\left(K^{3}\right)$ is the hypergeometric series

$$
G_{M}^{J}\left(K^{3}\right)={ }_{3} F_{2}\left(J, 2 j+J-1, J-M ; K^{3}+J+j-M, 2 J\right),
$$

defined by

$$
{ }_{3} F_{2}(a, b, c ; d, e)=\sum_{k=0}^{\infty} \frac{\Gamma(a+k) \Gamma(b+k) \Gamma(c+k) \Gamma(d) \Gamma(e)}{k ! \Gamma(a) \Gamma(b) \Gamma(c) \Gamma(d+k) \Gamma(e+k)} .
$$

To summarize, we have obtained explicit matrices $\Phi_{M}^{J}$ of the form

$$
\Phi_{M}^{J}=\left\{\begin{array}{l}
F_{M}^{J}\left(X^{3}\right) X^{+M}, M \geq 0 \\
\tilde{F}_{M}^{J}\left(X^{3}\right) X^{-M}, M \leq 0
\end{array},\right.
$$

\footnotetext{
${ }^{6}$ The solutions are degenerate in the case of $J$ being integer.
} 
realizing the decomposition (3.8) of $\mathcal{A}=\mathcal{H}_{j} \otimes \mathcal{H}_{j}^{*}$ into unitary representations of $\mathrm{SO}(2,1)$. They form an orthonormal basis for the inner product defined by the trace (3.5). This is in one-to-one correspondence with the decomposition (2.25) of classical functions

$$
\phi_{M}^{J}=\left\{\begin{array}{l}
f_{M}^{J}\left(x^{3}\right) x^{+M}, M \geq 0 \\
\tilde{f}_{M}^{J}\left(x^{3}\right) x^{-M}, M \leq 0
\end{array}\right.
$$

with $x^{+}=x^{1}+i x^{2}$ on the hyperboloid. Due to the relation with the Casimir, the spectrum of matrix d'Alembertian $\frac{1}{\kappa^{2}} \square$ in (3.9) and the classical d'Alembertian (2.20) coincide. Including also the space of polynomial functions $\operatorname{Pol}\left(X^{a}\right)$, this is the basis for interpreting the matrix algebra $\mathcal{A}$ as quantized algebra of functions over hyperboloid.

\subsection{Semi-classical limit}

Now consider the classical hyperboloid $\mathcal{M}$ as a Poisson manifold equipped with the Poisson structure (2.27). The quantization of such a Poisson manifold is defined in terms of a quantization map $\mathcal{Q}$, which is an isomorphism of vector spaces from the space of functions $\mathcal{C}(\mathcal{M})$ on $\mathcal{M}$ to some (operator) algebra $\mathcal{A}$

$$
\mathcal{Q}: \mathcal{C}(\mathcal{M}) \rightarrow \mathcal{A}, \quad f(x) \mapsto \mathcal{Q}(f(x))
$$

which is compatible with the Poisson structure $\{f, g\}=\theta^{\mu \nu} \partial_{\mu} f \partial_{\nu} g$, satisfying

$$
\begin{aligned}
\mathcal{Q}(f g)-\mathcal{Q}(f) \mathcal{Q}(g) & \rightarrow 0 \quad \text { and } \\
\frac{1}{\theta}(\mathcal{Q}(i\{f, g\})-[\mathcal{Q}(f), \mathcal{Q}(g)]) & \rightarrow 0 \quad \text { as } \quad \theta \rightarrow 0 .
\end{aligned}
$$

Clearly $\mathcal{Q} \equiv \mathcal{Q}_{\theta}$ depends on the Poisson structure $\theta$, and the limit $\theta \rightarrow 0$ is understood in some appropriate way; for a more mathematical discussion we refer e.g. to [33-36]. As $\mathcal{Q}$ is an isomorphism of vector spaces, ${ }^{7}$ one can then define the semi-classical limit of some fuzzy wavefunction $F \in \mathcal{A}$ as the inverse $f=\mathcal{Q}^{-1}(F)$ of the quantization map. This is consistent as $\theta \rightarrow 0$, provided commutators are replaced by the appropriate Poisson brackets, and higher order terms in $\theta$ are neglected.

In general, there is no unique way of defining $\mathcal{Q}$. However in the case at hand, there is a natural definition of $\mathcal{Q}$, based on the decomposition of $\mathcal{C}(\mathcal{M})$ and $\mathcal{A}$ into irreducible representations of $\mathrm{SO}(2,1)$. Given the corresponding orthonormal bases $\Phi_{M}^{J}$ and $\phi_{M}^{J}$ of $\mathcal{A}$ resp. $\mathcal{C}(\mathcal{M})$ as obtained above, we define

$$
\mathcal{Q}\left(\phi_{M}^{J}\right)=\Phi_{M}^{J},
$$

so that $\mathcal{Q}$ is an isometry for the unitary representations. This can be extended to the polynomials $\operatorname{Pol}\left(x^{a}\right)$, corresponding to finite-dimensional non-unitary irreducible representations of $\mathrm{SO}(2,1)$. However these are not normalizable, and the normalization of $\mathcal{Q}\left(\operatorname{Pol}\left(x^{a}\right)\right)$

\footnotetext{
${ }^{7}$ Sometimes one only requires $Q$ to be an isomorphism only on the space of functions with momenta below some UV cutoff.
} 
must be fixed in another way. Since we want to interpret the matrices $X^{a}, a=1,2,3$ as a quantized embedding coordinates $x^{a}, a=1,2,3$, we define

$$
\mathcal{Q}\left(x^{a}\right)=X^{a} .
$$

Comparing the embedding equation $x^{a} x_{a}=-R^{2}$ with the Casimir constraint $X^{a} X_{a}=$ $\kappa^{2} j(j-1)(3.2)$, we are led to impose

$$
\kappa^{2}\left(s^{2}+\frac{1}{4}\right)=R^{2}=\text { const }
$$

using $j=1 / 2+i s$ for the principal continuous representation $\mathcal{H}_{j}$. Therefore the semiclassical limit $\kappa \rightarrow 0$ implies $^{8} s \rightarrow \infty$. This is the analog of $N=\operatorname{dim} \mathcal{H} \rightarrow \infty$ in the case of fuzzy sphere.

To establish ${ }^{9}$ the required properties of $\mathcal{Q}$, we recall that all fuzzy wavefunctions can be written in the following "normal form" (3.25)

$$
\Phi_{M}^{J}=F_{M}^{J}\left(X^{3}\right) X^{+M}, \quad \phi_{M}^{J}=f_{M}^{J}\left(x^{3}\right) x^{+^{M}}, \quad M \geq 0
$$

and similarly for $M<0$. We claim that

$$
\lim _{\kappa \rightarrow 0} F_{M}^{J}=f_{M}^{J}
$$

as functions in one variable. To see this, observe that in the limit $\kappa \rightarrow 0$ following relations hold

$$
\begin{aligned}
\lim _{\kappa \rightarrow 0} \frac{1}{\kappa}\left[X^{ \pm}, F\left(X^{3}\right)\right] & =\mp F^{\prime}\left(X^{3}\right) X^{ \pm}, \\
\lim _{\kappa \rightarrow 0} \frac{1}{\kappa}\left[X^{-}, X^{+M}\right] & =2 M X^{3} X^{+M-1},
\end{aligned}
$$

as a consequence of the Lie algebra relations. In the classical case, the corresponding relations are

$$
\begin{aligned}
i\left\{X^{ \pm}, f\left(x^{3}\right)\right\} & =\mp f^{\prime}\left(x^{3}\right) x^{ \pm}, \\
i\left\{x^{-}, x^{+M}\right\} & =2 M x^{3} x^{+M-1} .
\end{aligned}
$$

Therefore the action of the matrix Laplacian (3.9) on $\Phi_{M}^{J}$ in the limit $\kappa \rightarrow 0$

$$
\begin{aligned}
& \lim _{\kappa \rightarrow 0} \frac{1}{\kappa^{2}} \square \Phi_{M}^{J}= \\
& \quad-\left[\left(X^{3^{2}}+R^{2}\right){F^{\prime \prime}}_{M}^{J}\left(X^{3}\right)+2(M+1) X^{3}{F^{\prime}}_{M}^{J}\left(X^{3}\right)+M(M+1) F_{M}^{J}\left(X^{3}\right)\right] X^{+M}(3.3)
\end{aligned}
$$

has precisely the same form as the action of the classical Laplacian

$$
R^{2} \square_{g} \phi_{M}^{J}=-\frac{1}{\kappa^{2}}\left\{x^{+},\left\{x^{-}, \phi_{M}^{J}\right\}\right\}+\frac{1}{\kappa^{2}}\left\{x^{3},\left\{x^{3}, \phi_{M}^{J}\right\}\right\}+\frac{i}{\kappa}\left\{x^{3}, \phi_{M}^{J}\right\}=-J(J-1) \phi_{M}^{J} .
$$

\footnotetext{
${ }^{8}$ This is also the reason why the complementary series has been rejected for $\mathcal{H}_{j}$.

${ }^{9}$ Our aim is to establish and clarify the required properties, without claiming mathematical rigor.
} 
This implies (3.34) up to normalization, and allows to define $\mathcal{Q}$ in such a way that $\mathcal{Q}\left(f_{M}^{J}\left(x^{3}\right) x^{+M}\right) \rightarrow f_{M}^{J}\left(X^{3}\right) X^{+M}$ as $\kappa \rightarrow 0$. In particular, this provides the appropriate definition of $\mathcal{Q}$ for the principal continuous representation $P_{S}$ (which is doubly degenerate), as well as for the finite-dimensional polynomials which are not normalizable.

Now it is easy to see that $\mathcal{Q}$ respects the algebra structure and the Poisson bracket in the limit $\kappa \rightarrow 0$. Consider the product of two matrix modes as above, expanded up to leading order in $\kappa$

$$
\begin{aligned}
\mathcal{Q}\left(\Phi_{M}^{J}\right) \mathcal{Q}\left(\Phi_{M^{\prime}}^{J^{\prime}}\right) & =\Phi_{M}^{J} \Phi_{M^{\prime}}^{J^{\prime}}=F_{M}^{J} X^{+M} F_{M^{\prime}}^{J^{\prime}} X^{+M^{\prime}}=F_{M}^{J}\left[X^{+M}, F_{M^{\prime}}^{J^{\prime}}\right] X^{+M^{\prime}}+F_{M}^{J} F_{M^{\prime}}^{J^{\prime} X^{+}} \\
& =\left(F_{M}^{J} F_{M^{\prime}}^{J^{\prime}}-M \kappa F_{M}^{J}{F^{\prime}}_{M^{\prime}}^{J^{\prime}}+o\left(\kappa^{2}\right)\right) X^{+M+M^{\prime}}
\end{aligned}
$$

for $M, M^{\prime} \geq 0$. Then (3.28) follows immediately using (3.34). Subtracting the same computation with the factors reversed, (3.29) follows. A similar computation applies to modes with mixed or negative $M$. Therefore $\mathcal{Q}$ is indeed a quantization map for our Poisson structure. Using the decomposition of $\mathcal{A}$ into the above modes, analogous statements can be made for the de-quantization map $\mathcal{Q}^{-1}$, which provides the semi-classical limit of the fuzzy wavefunctions.

Finally, consider the trace of some normalizable wavefunctions with weight $M=0$,

$$
\begin{aligned}
2 \pi \operatorname{Tr} \Phi_{0}^{J^{\dagger}} \Phi_{0}^{J} & =2 \pi \sum_{m=-\infty}^{\infty} F_{0}^{J *}(\kappa m) F_{0}^{J}(\kappa m) \stackrel{\kappa \rightarrow 0}{\rightarrow} 2 \pi \kappa^{-1} \int d x^{3} f_{0}^{J^{*}}\left(x^{3}\right) f_{0}^{J}\left(x^{3}\right) \\
& =\int \omega \phi_{0}^{* J} \phi_{0}^{J},
\end{aligned}
$$

using the explicit form of $\mathcal{H}_{j}=P_{s}$ (2.18), where $\omega$ is the symplectic form (2.26). This computation is easily generalized to show that

$$
2 \pi \operatorname{Tr} \mathcal{Q}(f) \mathcal{Q}(g) \stackrel{\kappa \rightarrow 0}{\rightarrow} \int \omega f g
$$

as long as the integrals are bounded. This is guaranteed for the spaces of unitary wavefunctions discussed above.

To summarize, in the semiclassical limit $\sim$ defined as de-quantization map expanded up to leading order in $\kappa$, we can use the following relations

$$
\Phi_{M}^{J} \sim \phi_{M}^{J}, X^{a} \sim x^{a},[F, G] \sim i\left\{\mathcal{Q}^{-1}(F), \mathcal{Q}^{-1}(G)\right\},\left[X^{a},\right] \sim i\left\{x^{a},\right\}, 2 \pi \operatorname{Tr} \sim \int \omega
$$

which we use in the following sections.

\section{Dynamical matrix models}

Consider now three hermitian matrices $X^{a}=\left(X^{a}\right)^{\dagger} \in \operatorname{Mat}(\infty, \mathbb{C})$ for $a=1,2,3$, which transform in the basic 3 -dimensional representation of $\mathrm{SO}(2,1)$. Then the most general matrix model up to order 4 which is invariant under the $\mathrm{SO}(2,1)$ symmetry as well as translations $X^{a} \rightarrow X^{a}+c^{a} \mathbb{1}$ has the form

$$
S[X]=-\frac{2 \pi}{g_{Y M}^{2}} \operatorname{Tr}\left(\left[X^{a}, X^{b}\right]\left[X_{a}, X_{b}\right]+i g_{3} f_{a b c}\left[X^{a}, X^{b}\right] X^{c}\right)
$$


for suitable constants, where embedding indices are raised and lowered with $\eta_{a b}$. The matrices $X^{a}$ are understood to have dimension length, and accordingly $\left[g_{Y M}\right]=L^{2}$. This model is invariant under $\mathrm{SO}(2,1)$ rotations, translations as well as gauge transformations $X^{a} \rightarrow U X^{a} U^{-1}$ for unitary operators $U$. The equations of motion are obtained as

$$
\begin{aligned}
4 \square X^{a} & =3 i g_{3} f_{b c}^{a}\left[X^{b}, X^{c}\right], \\
\square & \equiv\left[X^{a},\left[X_{a}, .\right]\right] .
\end{aligned}
$$

Now consider the ansatz ${ }^{10}$

$$
X^{a}=\kappa K^{a}, \quad a=1,2,3
$$

in terms of rescaled generators of a unitary irreducible representation of $\mathrm{SO}(2,1)$. Then

$$
\begin{aligned}
{\left[X^{a}, X^{b}\right] } & =i \kappa f_{c}^{a b} X^{c}, \\
X^{a} X_{a} & =\left.\kappa^{2} C\right|_{\mathcal{H}}=-\kappa^{2}\left(s^{2}+\frac{1}{4}\right) \mathbb{1}_{\mathcal{H}}=-R^{2} \mathbb{1}_{\mathcal{H}}, \\
\square X^{a} & =\left.\kappa^{2} C\right|_{a d} X^{a}=2 \kappa^{2} X^{a}
\end{aligned}
$$

where $C$ is the quadratic Casimir of $\mathrm{SO}(2,1)$, and $\kappa, R$ are positive numbers. As discussed before, we take $\mathcal{H}=\mathcal{H}_{j}$ to be the principal continuous series representation, so that $X^{a} \in \operatorname{End}(\mathcal{H})$ and

$$
\frac{R^{2}}{\kappa^{2}}=\left(s^{2}+\frac{1}{4}\right)=-\left.C\right|_{\mathcal{H}} .
$$

Thus the equations of motion (4.2) are solved by this ansatz provided

$$
4 \kappa^{2}+3 \kappa g_{3}=0
$$

This is a quadratic equation in $\kappa$ which we assume to have a positive solution.

The effective matrix geometry. Let us discuss the geometry of the fuzzy brane solutions in the matrix model in the semi-classical limit, following $[19,20]$. Recall that the matrices $X^{a}$ are interpreted as quantized Cartesian embedding functions

$$
X^{a} \sim x^{a}: \quad \mathcal{M} \hookrightarrow \mathbb{R}^{3}
$$

The induced metric on $\mathcal{M}$ is given by

$$
g_{\mu \nu}=\partial_{\mu} x^{a} \partial_{\nu} x_{a}
$$

For the hyperboloid solutions under consideration $x^{a} x_{a}=-R^{2}$ holds, so that the induced metric is that of $A d S^{2}$. However, we are interested in the effective metric which governs

\footnotetext{
${ }^{10}$ Of course the matrix model action is divergent on this background, however this is not a problem. We only need to require that the perturbations lead to a finite variation of the action. This could be taken into account by subtracting certain background terms, which we do not write down for brevity.
} 
physical fields in the matrix model. To identify the effective metric in the semi-classical limit, we note that the kinetic term e.g. for a scalar field $\Phi$ in the matrix model ${ }^{11}$ arises from an action of the form

$$
\begin{aligned}
S[\phi] & =-\frac{2 \pi}{2 g_{Y M}^{2}} \operatorname{Tr}\left[X_{a}, \Phi\right]\left[X^{a}, \Phi\right] \sim \frac{1}{2 g_{Y M}^{2}} \int \omega \theta^{\mu \mu^{\prime}} \theta^{\nu \nu^{\prime}} g_{\mu^{\prime} \nu^{\prime}} \partial_{\mu} \phi \partial_{\nu} \phi \\
& =-\frac{1}{2 g_{Y M}} \int d^{2} x \sqrt{|G|} e^{-\sigma / 2} G^{\mu \nu} \partial_{\mu} \phi \partial_{\nu} \phi=-\frac{1}{2} \int d^{2} x \sqrt{|G|} e^{-\sigma / 2} G^{\mu \nu} \partial_{\mu} \varphi \partial_{\nu} \varphi,
\end{aligned}
$$

using the semi-classical correspondence rules (3.44). Here the scalar fields are made dimensionless via $\Phi \sim \phi=g_{Y M}^{1 / 2} \varphi$, and

$$
\begin{aligned}
\omega & =\frac{1}{2} \theta_{\mu \nu}^{-1} d x^{\mu} d x^{\nu}, \\
G^{\mu \nu} & =-g_{Y M}^{-2} \theta^{\mu \mu^{\prime}} \theta^{\nu \nu^{\prime}} g_{\mu^{\prime} \nu^{\prime}}=e^{-\sigma} g^{\mu \nu}, \\
e^{-\sigma} & =g_{Y M}^{-2}\left|\operatorname{det} \theta^{\mu \nu}\right|\left|\operatorname{det} g_{\mu \nu}\right| .
\end{aligned}
$$

Therefore scalar fields couple to the effective metric given by $G^{\mu \nu}$. Note the explicit minus in the definition of $G^{\mu \nu}$, which is in contrast to the higher-dimensional case discussed in $[19,20]$. The correct sign can be obtained from the action (4.1) resp. (4.9), which must have the form $S=\int d t(T-V)$. For the action (4.1) it means that the effective metric is indeed that of $A d S^{2}$, while fuzzy $d S^{2}$ can be obtained by changing the overall sign of the action. This freedom to change the sign is possible only in the case of signature $(-+)$ in 2 dimensions. Note also that for 2-dimensional branes, the conformal factor of the effective metric is not fixed by the above scalar field action, due to the Weyl symmetry $G^{\mu \nu} \rightarrow e^{\alpha} G^{\mu \nu}$. Here we choose (4.10) for simplicity; our main goal is to illustrate how such an effective metric responds to matter perturbations in the present matrix model.

The relation $G \sim g$ is particular for 2 dimensions, and can be seen in coordinates where $g_{\mu \nu}=\operatorname{diag}(-1,1)$ at some given point $p \in \mathcal{M}^{2}$. Consider the point $p_{N}=(R, 0,0)$ in the homogeneous $A d S^{2}$ space. Its tangent space is parallel to the $\left(x^{2} x^{3}\right)$ plane, so that we can use $x^{\mu}=\left(x^{2}, x^{3}\right)$ as local coordinates. In these "normal embedding" coordinates we have $g_{\mu \nu}=\operatorname{diag}(-1,1)$ at $p_{N}$, and $\theta^{23}=\left\{x^{2}, x^{3}\right\}=\kappa f_{1}^{23} R=\kappa R$. On the other hand $\theta^{\mu \nu}=\left\{x^{\mu}, x^{\nu}\right\}=g_{Y M} e^{-\sigma / 2} \epsilon^{\mu \nu}$ using (4.10), and we obtain

$$
e^{-\sigma / 2}=\left|g_{Y M}^{-1} x^{1}\right|=g_{Y M}^{-1} \kappa R
$$

We note that the matrix Laplace operator (4.4) for the unperturbed hyperboloid background is related to the geometric Laplace operator in the semi-classical limit ${ }^{12}$

$$
\square \Phi \sim-\left\{x^{a},\left\{x_{a}, \phi\right\}\right\}=g_{Y M}^{2} \frac{1}{\sqrt{G}} \partial_{\mu}\left(\sqrt{G} G^{\mu \nu} \partial_{\nu} \phi\right)=g_{Y M}^{2} \square_{G} \phi .
$$

\footnotetext{
${ }^{11}$ For example, the radial components of the non-abelian fluctuations on a stack of coincident branes realize such scalar fields. A detailed analysis of general abelian perturbations will be given below.

${ }^{12}$ Although such a relation holds very generally in the higher-dimensional case [19, 20], it is restricted to $e^{\sigma}=$ const in 2 dimensions; for a general formula in 2 dimensions see [37]. Here we need the Laplacian only for the unperturbed backgrounds, where (4.12) is sufficient.
} 
Finally, it is easy to add fermions the matrix model, via the action

$$
S[\psi]=\operatorname{Tr} \bar{\Psi}\left(\not D+m_{\psi}\right) \Psi .
$$

Here $\not D \Psi=\Gamma_{a}\left[X^{a}, \Psi\right]$ is the matrix Dirac operator, and

$$
\Psi=\left(\begin{array}{c}
\psi_{1} \\
\psi_{2}
\end{array}\right), \quad \psi_{\alpha} \in \mathcal{A}
$$

is a 2-component spinors of $\mathrm{SO}(2,1)$, and $\Gamma_{a}$ satisfy the Clifford algebra of $\mathrm{SO}(2,1)$,

$$
\Gamma_{a} \Gamma_{b}+\Gamma_{b} \Gamma_{a}=2 \eta_{a b} .
$$

In the semi-classical limit, the Dirac operator can be written as

$$
\not D \Psi=\Gamma_{a}\left[X^{a}, \Psi\right] \sim i \tilde{\gamma}^{\mu} D_{\mu}
$$

where the $\tilde{\gamma}^{\nu}=\theta^{\nu \mu} \partial_{\nu} x^{a} \Gamma_{a}$ satisfy the Clifford algebra associated with the effective metric

$$
\left\{\tilde{\gamma}^{\mu}, \tilde{\gamma}^{\nu}\right\}=2 G^{\mu \nu}
$$

This implies that the fermions also couple to the effective metric $G^{\mu \nu}$.

In general, any physical field in the matrix model with background $X^{a}$ as above arises either as fluctation of the matrices around the background, or from the fermionic matrices $\Psi$. In any case, it can be interpreted in terms of functions on the brane $\mathcal{M}$. Since derivatives of these fields can only arise from commutators with the background matrices, the kinetic term for bosonic fields arises from a double commutator term, which is governed by $G^{\mu \nu}$ in the semi-classical limit as shown above. The same conclusion holds for non-abelian gauge fields and fermions, as established in more detail in [19, 20]. Therefore $G^{\mu \nu}$ should be identified as gravitational metric on the brane. Gauge transformations in the matrix model correspond to symplectomorphisms in the semi-classical limit, which form a subgroup of the diffeomorphism group, and can be gauge-fixed as in a U(1) gauge theory. The full diffeomorphism group plays no role in the matrix model, since the geometry arises in a partially gauge-fixed way (essentially in harmonic gauge with respect to $x^{\mu} \sim X^{\mu}$, cf. [19, 20]) on sub-manifolds embedded in an un-physical higher-dimensional Minkowski space. In the semi-classical limit of course, one may express the geometry in any coordinates, thus re-introducing a redundant diffeomorphism invariance.

\section{$5 \quad$ Fluctuating $A d S^{2}$ and gravity}

We introduce some useful geometrical structures which apply to general $\mathcal{M}^{2} \subset \mathbb{R}^{3}$. The "translational currents"

$$
J_{\mu}^{a}=\partial_{\mu} x^{a}
$$

span the tangent space of $\mathcal{M}^{2} \subset \mathbb{R}^{3}$, while

$$
K_{\mu \nu}^{a}=\nabla[g]_{\mu} J_{\nu}^{a}=K_{\nu \mu}^{a}
$$


characterizes the extrinsic curvature and is normal to the brane with respect to the embedding metric,

$$
J_{\mu}^{a} K_{a \nu \eta}=0
$$

In particular,

$$
K^{a}=K_{\mu \nu}^{a} G^{\mu \nu}=\square_{G} x^{a}
$$

is a normal vector ${ }^{13}$ to $\mathcal{M}^{2} \subset \mathbb{R}^{3}$. For the present $A d S^{2}$ solution, one can easily compute the curvature of the connection $\nabla[G]=\nabla[g] \equiv \nabla$,

$$
\begin{aligned}
K_{\mu \nu}^{a} & =R^{-2} g_{\mu \nu} x^{a}=\frac{1}{2} G_{\mu \nu} K^{a}, \\
K^{a} & =2 e^{-\sigma} R^{-2} x^{a}=-\operatorname{Ric}[G] x^{a}
\end{aligned}
$$

This is consistent with $\square_{g} x^{a}=\frac{2}{R^{2}} x^{a}$ on $A d S^{2}$. The Riemann curvature tensor can be obtained e.g. from the Gauss-Codazzi theorem, and is given by

$$
\begin{aligned}
\mathrm{R}_{\mu \nu \eta}{ }^{\rho} & =-R^{-2}\left(g_{\mu \eta} \delta_{\nu}^{\rho}-g_{\nu \eta} \delta_{\mu}^{\rho}\right), \\
\operatorname{Ric}_{\mu \nu} & =\frac{1}{2} \operatorname{Ric}[g] g_{\mu \nu}=\frac{1}{2} \operatorname{Ric}[G] G_{\mu \nu}, \\
\operatorname{Ric}[g] & =-2 R^{-2}, \quad \operatorname{Ric}[G]=-2 R^{-2} e^{-\sigma}
\end{aligned}
$$

using (4.11), and recalling $x^{a} x_{a}=-R^{2}$. Using the above relations along with (4.4), the embedding functions $x^{a}$ satisfy

$$
\left(\square_{G}+\operatorname{Ric}[G]\right) x^{a}=0
$$

Now consider small fluctuations around the solutions $\bar{X}^{a}$ of the above matrix model, parametrized as

$$
X^{a}=\bar{X}^{a}+\mathcal{A}^{a}(\bar{X})
$$

These fluctuations can be interpreted in different ways. First, one can decompose the $\mathcal{A}^{a}$ into tangential and one radial components, analogous to the well-known case of the fuzzy sphere [7]. Then the radial component can be interpreted as scalar field on $\mathcal{M}^{2}$, and the tangential components in terms of (noncommutative) gauge fields. This interpretation is certainly appropriate for the non-abelian components, which arise on a stack of $n$ coinciding such branes. However since the trace- U(1) components change the effective metric $G^{\mu \nu}$ on $\mathcal{M}^{2}$, it is more natural to interpret them in terms of geometrical or gravitational degrees of freedom; note that there is no charged object under this $\mathrm{U}(1)$. In this section we elaborate some aspects of the resulting 2-dimensional effective or "emergent" gravity. ${ }^{14}$

\footnotetext{
${ }^{13}$ In general, this holds for $\square_{g}$ rather than $\square_{G}$, but in the 2-dimensional case both statements are true.

${ }^{14}$ The word "emergent" indicates that the metric arises from other, more fundamental degrees of freedom.
} 
In the semi-classical limit, the matrix model action expanded to second order in $\mathcal{A}^{a}$ around the basic $A d S^{2}$ solution is given by

$$
\begin{aligned}
S[X] & \sim \frac{2}{g_{Y M}^{2}} \int \omega\left(\left\{x^{a}, \mathcal{A}^{b}\right\}\left\{x_{a}, \mathcal{A}_{b}\right\}+\left\{x^{a}, \mathcal{A}^{b}\right\}\left\{\mathcal{A}_{a}, x_{b}\right\}+\left(\kappa+\frac{3 g_{3}}{2}\right) f_{a b c}\left\{\mathcal{A}^{a}, \mathcal{A}^{b}\right\} x^{c}\right) \\
& =\frac{2}{g_{Y M}^{2}} \int \omega\left(g_{Y M}^{2} \mathcal{A}_{b} \square_{G} \mathcal{A}^{b}-f^{2}+\left(2 \kappa+\frac{3 g_{3}}{2}\right) f_{a b c} x^{c}\left\{\mathcal{A}^{a}, \mathcal{A}^{b}\right\}\right) \\
& =\frac{2}{g_{Y M}^{2}} \int \omega\left(g_{Y M}^{2} \mathcal{A}_{b} \square_{G} \mathcal{A}^{b}-f^{2}\right)
\end{aligned}
$$

dropping the linear as well as the $f_{a b c}$ term which vanish due to the equations of motion (4.6), and using

$$
\begin{aligned}
\int\left\{x^{a}, \mathcal{A}^{b}\right\}\left\{\mathcal{A}_{a}, x_{b}\right\} & =-\int \mathcal{A}^{b}\left\{x^{a},\left\{\mathcal{A}_{a}, x_{b}\right\}\right\}=\int \mathcal{A}^{b}\left(\left\{\mathcal{A}_{a},\left\{x_{b}, x_{a}\right\}\right\}+\left\{x_{b},\left\{x_{a}, \mathcal{A}_{a}\right\}\right\}\right\} \\
& =\int\left(\kappa f_{a b c}\left\{\mathcal{A}^{a}, \mathcal{A}^{b}\right\} x^{c}+\left\{\mathcal{A}^{b}, x_{b}\right\},\left\{x_{a}, \mathcal{A}^{a}\right\}\right)
\end{aligned}
$$

Here

$$
f=\left\{\mathcal{A}^{a}, x_{a}\right\}
$$

can be viewed as gauge fixing function, since it transforms as

$$
f \rightarrow f+\left\{x_{a},\left\{x^{a}, \Lambda\right\}\right\}=f-g_{Y M}^{2} \square_{G} \Lambda
$$

under gauge transformations. We can thus choose the gauge such that $f=0$.

We want to understand how the geometry is influenced by matter. We assume that all fields on $\mathcal{M}^{2}$ couple to the effective metric ${ }^{15} G_{\mu \nu}$, so that the metric perturbations couple to matter via the energy-momentum tensor. The linearized metric fluctuation is given by

$$
\begin{aligned}
\delta_{\mathcal{A}} g_{\mu \nu} & =J_{\mu}^{a} \partial_{\nu} \mathcal{A}_{a}+J_{\nu}^{a} \partial_{\mu} \mathcal{A}_{a} \\
& =\nabla_{\mu} \mathcal{A}_{\nu}+\nabla_{\nu} \mathcal{A}_{\mu}-2 K_{\mu \nu}^{a} \mathcal{A}_{a}
\end{aligned}
$$

where we decompose the perturbations into tangential and transversal ones

$$
\mathcal{A}^{\perp}=K^{a} \mathcal{A}_{a}, \quad \mathcal{A}_{\mu}=J_{\mu}^{a} \mathcal{A}_{a} .
$$

Using $2 K_{\mu \nu}^{a}=e^{\sigma} g_{\mu \nu} K^{a}(5.5)$, the perturbation of the effective metric in Darboux coordinates can be written as

$$
\begin{aligned}
\delta_{\mathcal{A}} G^{\mu \nu} & =-g_{Y M}^{-2} \theta^{\mu \mu^{\prime}} \theta^{\nu \nu^{\prime}} \delta_{\mathcal{A}} g_{\mu \nu} \\
& =-g_{Y M}^{-2} \theta^{\mu \mu^{\prime}} \theta^{\nu \nu^{\prime}}\left(\nabla_{\mu} \mathcal{A}_{\nu}+\nabla_{\nu} \mathcal{A}_{\mu}\right)-e^{\sigma} G^{\mu \nu} K^{a} \mathcal{A}_{a}
\end{aligned}
$$

\footnotetext{
${ }^{15}$ We ignore possibly different conformal factors for different types of matter, for the sake of illustrating the mechanism in a simple toy model.
} 
Therefore

$$
\delta_{\mathcal{A}} S_{M}=-\frac{1}{2} \int d^{2} x \sqrt{G} T_{\mu \nu} \delta_{\mathcal{A}} G^{\mu \nu}=\int d^{2} x \sqrt{G}\left(\frac{e^{\sigma}}{2} T K^{a}-\nabla_{\mu} \tilde{T}^{\mu \nu} J_{\nu}^{a}\right) \mathcal{A}_{a}
$$

noting that $\nabla \theta^{\mu \nu}=0$, where $T=T_{\mu \nu} G^{\mu \nu}$, and we define

$$
\tilde{T}^{\mu \nu}=g_{Y M}^{-2} \theta^{\nu \nu^{\prime}} \theta^{\mu \mu^{\prime}} T_{\mu^{\prime} \nu^{\prime}}
$$

for convenience. Thus the normal component $\mathcal{A}^{\perp}$ couples to the trace of the energymomentum tensor, while the tangential components couple to its derivative. This illustrates the observation $[21,22]$ that a non-derivative coupling of the embedding perturbations to the energy-momentum tensor arises on branes with extrinsic curvature. Using the on-shell condition (4.6) for the background and

$$
\sqrt{\left|G_{\mu \nu}\right|\left|\theta^{\mu \nu}\right|}=g_{Y M} e^{\sigma / 2}
$$

we obtain the semi-classical equations of motion

$$
\square_{G} \mathcal{A}^{a}=\frac{1}{8} g_{Y M} e^{\sigma / 2}\left(-e^{\sigma} T K^{a}+2 J_{\mu}^{a} \nabla_{\nu} \tilde{T}^{\mu \nu}\right) .
$$

Note that the normal component couples to $T$ via the extrinsic curvature. This is the crucial ingredient for gravity, as we will see below.

\subsection{Curvature perturbations and gravity}

Now we can obtain the curvature perturbations induced by matter. Since in 2 dimensions $\operatorname{Ric}_{\mu \nu}[G]=\frac{1}{2} G_{\mu \nu} \operatorname{Ric}[G]$ where $\operatorname{Ric}[G]$ is the Ricci scalar, we will restrict ourselves to study the linearized perturbations of Ric $[G]$. This can be computed using

$$
\delta R_{\mu \nu}[G]=-\frac{1}{2} \nabla_{\mu} \partial_{\nu}\left(G^{\rho \eta} \delta G_{\rho \eta}\right)-\frac{1}{2} \square_{G} \delta G_{\mu \nu}+\nabla_{(\mu} \nabla^{\eta} \delta G_{\nu) \eta}
$$

which implies

$$
\delta_{\mathcal{A}} \operatorname{Ric}[G]=\left(\square_{G}+\frac{1}{2} \operatorname{Ric}[G]\right)\left(G_{\mu \nu} \delta_{\mathcal{A}} G^{\mu \nu}\right)-\nabla_{\mu} \nabla_{\nu} \delta_{\mathcal{A}} G^{\mu \nu}
$$

The perturbation of the effective metric in Darboux coordinates can be written as follows (cf. (5.15))

$$
\delta_{\mathcal{A}} G^{\mu \nu}=-g_{Y M}^{-2} \theta^{\mu \mu^{\prime}} \theta^{\nu \nu^{\prime}}\left(J_{\mu^{\prime}}^{a} \partial_{\nu^{\prime}} \mathcal{A}_{a}+J_{\nu^{\prime}}^{a} \partial_{\mu^{\prime}} \mathcal{A}_{a}\right)
$$

using $\delta \theta^{\mu \nu}=0$. After some computations given in the appendix, the corresponding perturbation of the Ricci tensor is obtained as

$$
\begin{aligned}
\delta_{\mathcal{A}} \operatorname{Ric}[G] & =\frac{1}{2} g_{Y M} e^{\sigma / 2}\left(R^{-2} T+\nabla_{\mu} \nabla_{\nu} \tilde{T}^{\mu \nu}\right) \\
& =8 \pi G_{N}\left(T+R^{2} \nabla_{\mu} \nabla_{\nu} \tilde{T}^{\mu \nu}\right), \\
8 \pi G_{N} & =e^{\sigma / 2} \frac{g_{Y M}}{2 R^{2}}=\frac{\kappa e^{\sigma}}{2 R} .
\end{aligned}
$$


This can be seen as linearization of the following gravity model

$$
\begin{aligned}
\operatorname{Ric}[G]-\Lambda & =8 \pi G_{N} T+\mathcal{O}(\partial \partial T), \\
\Lambda & =-2 e^{-\sigma} R^{-2}
\end{aligned}
$$

which is reasonable and non-trivial in 2 dimensions [24, 25] (dropping the $\mathcal{O}(\partial \partial T)$ terms), unlike general relativity which does not allow any coupling to matter. Note that the derivative term is of order

$$
\nabla \nabla \tilde{T} \sim e^{\sigma} \nabla \nabla T
$$

using (5.18), and can be neglected provided $e^{\sigma} \ll 1$, which is compatible with $G_{N} \ll 1$. Although we focused on the $A d S^{2}$ background, the result should equally apply to the $d S^{2}$ background, which is obtained by changing the sign of the matrix model action.

We emphasize again that no specific gravity action was assumed or induced, we have simply elaborated the matrix model dynamics from a geometrical point of view. The crucial coupling to $T_{\mu \nu}$ arises due to the extrinsic curvature of the brane encoded in $\nabla_{\mu} J_{\nu}^{a}=K_{\mu \nu}^{a}$, as pointed out in [21, 22]; this is already seen in (5.19). Also, it is gratifying (and not evident) that the Newton constant turns out to be positive. The mechanism is basically the same as the "gravity bag" mechanism discussed in [23]. Its 4-dimensional version is clearly more complicated and currently under investigation, however at least certain aspects of the mechanism generalize [21, 22].

However, since the gravitational coupling is dynamical itself, the above linearized treatment of the coupling is justified only as long as the perturbations of the radial $K_{\mu \nu}^{a}$ is negligible, i.e.

$$
\delta K_{\mu \nu}^{a} \ll K_{\mu \nu}^{a}
$$

For the $A d S^{2}$ backgrounds under consideration, this implies that the intrinsic curvature perturbation is smaller than the background constant curvature. This is clearly inadequate for physical gravity, however the basic mechanism should extend beyond this regime for backgrounds where the extrinsic curvature dominates the intrinsic one, such as cylinders or generalizations.

\subsection{Induced metric curvature}

It is instructive to compute also the Ricci tensor for the induced metric $g_{\mu \nu}$. Recall the decomposition of $\mathcal{A}_{a}$ into tangential and normal components (5.14). We have

$$
\delta_{\mathcal{A}} \operatorname{Ric}[g]=-\left(\square_{g}+\frac{1}{2} \operatorname{Ric}[g]\right)\left(g^{\mu \nu} \delta_{\mathcal{A}} g_{\mu \nu}\right)+\nabla^{\mu} \nabla^{\nu} \delta_{\mathcal{A}} g_{\mu \nu} .
$$

Writing the metric perturbation as

$$
\delta_{\mathcal{A}} g_{\mu \nu}=\nabla_{\mu} \mathcal{A}_{\nu}+\nabla_{\nu} \mathcal{A}_{\mu}-2 R^{-2} g_{\mu \nu} x^{a} \mathcal{A}_{a}
$$


one finds

$$
\begin{aligned}
\nabla^{\mu} \nabla^{\nu} \delta_{\mathcal{A}} g_{\mu \nu} & =\nabla^{\mu} \nabla^{\nu}\left(\nabla_{\mu} \mathcal{A}_{\nu}+\nabla_{\nu} \mathcal{A}_{\mu}-2 R^{-2} g_{\mu \nu} x^{a} \mathcal{A}_{a}\right) \\
& =2 \square_{g}\left(\nabla^{\nu} \mathcal{A}_{\nu}\right)+\operatorname{Ric}[g]\left(\nabla^{\nu} \mathcal{A}_{\nu}\right)-2 R^{-2} \square_{g}\left(x^{a} \mathcal{A}_{a}\right),
\end{aligned}
$$

noting that $\operatorname{Ric}_{\mu \nu}[g]=\frac{1}{2} \operatorname{Ric}[g] g_{\mu \nu}$ and the identity (5.37). Therefore

$$
\begin{aligned}
\delta_{\mathcal{A}} \operatorname{Ric}[g]= & -\left(2 \square_{g}+\operatorname{Ric}[g]\right)\left(\nabla^{\nu} \mathcal{A}_{\nu}-2 R^{-2} x^{a} \mathcal{A}_{a}\right) \\
& +2 \square_{g}\left(\nabla^{\nu} \mathcal{A}_{\nu}\right)+\operatorname{Ric}[g]\left(\nabla^{\nu} \mathcal{A}_{\nu}\right)-2 R^{-2} \square_{g}\left(x^{a} \mathcal{A}_{a}\right) \\
= & 2 R^{-2}\left(\square_{g}+\operatorname{Ric}[g]\right)\left(x^{a} \mathcal{A}_{a}\right) .
\end{aligned}
$$

As a consistency check, we note that the tangential variations $\mathcal{A}_{\mu}$ drop out, since they correspond to a diffeomorphism. Since $g=e^{\sigma} G$, this is related to $\delta_{\mathcal{A}} \operatorname{Ric}[G]$ up to conformal rescaling contributions.

\subsection{Gauge theory point of view}

In this final section, we disentangle and essentially solve the model using the gauge theory point of view. Recall the decomposition (5.14) of $\mathcal{A}^{a}$ into normal and tangential components. For the normal perturbations $\mathcal{A}^{\perp}$, we can use the identity

$$
\begin{aligned}
\square_{G} \mathcal{A}^{\perp} & =-\operatorname{Ric}[G]\left(K^{a} A_{a}\right)+2 \nabla_{\mu} K^{a} \partial^{\mu} \mathcal{A}_{a}+K^{a} \square_{G} A_{a} \\
& =\operatorname{Ric}[G] \mathcal{A}^{\perp}-2 \operatorname{Ric}\left(\nabla^{\mu} \mathcal{A}_{\mu}\right)+K^{a} \square_{G} A_{a},
\end{aligned}
$$

so that using the equation of motion (A.9) gives

$$
\square_{G} \mathcal{A}^{\perp}=\operatorname{Ric} \mathcal{A}^{\perp}-2 \operatorname{Ric}\left(\nabla^{\mu} \mathcal{A}_{\mu}\right)+\frac{1}{2} \kappa R^{-1} T .
$$

Similarly, consider the divergence of the tangential perturbations

$$
\nabla^{\mu} \mathcal{A}_{\mu}=\nabla^{\mu}\left(J_{\mu}^{a} \mathcal{A}_{a}\right)=K^{a} \mathcal{A}_{a}+J_{\mu}^{a} \nabla^{\mu} \mathcal{A}_{a} .
$$

The tangential components of the equation of motion give

$$
J_{\mu}^{a} \square_{G} \mathcal{A}_{a}=\frac{1}{4} g_{Y M} e^{\sigma / 2} g_{\eta \mu} \nabla_{\nu} \tilde{T}^{\eta \nu},
$$

so that

$$
\begin{aligned}
\square_{G} \mathcal{A}_{\mu} & =2 \nabla_{\rho} J_{\mu}^{a} \partial^{\rho} \mathcal{A}_{a}+\square_{G} J_{\mu}^{a} \mathcal{A}_{a}+\frac{1}{4} g_{Y M} e^{\sigma / 2} g_{\eta \mu} \nabla_{\nu} \tilde{T}^{\eta \nu} \\
& =2 K_{\rho \mu}^{a} \partial^{\rho} \mathcal{A}_{a}-\frac{1}{2} \operatorname{Ric}[G] \mathcal{A}_{\mu}+\frac{1}{4} g_{Y M} e^{-\sigma / 2} G_{\eta \mu} \nabla_{\nu} \tilde{T}^{\eta \nu}
\end{aligned}
$$

using $\nabla^{\mu} K_{\mu \nu}^{a}=\square_{G} J_{\mu}^{a}=-\frac{1}{2} \operatorname{Ric}[G] J_{\mu}^{a}$ and $K_{\rho \mu}^{a}=\frac{1}{2} G_{\rho \mu} K^{a}$. This gives

$$
\begin{aligned}
\nabla^{\mu} \square_{G} \mathcal{A}_{\mu} & =-\operatorname{Ric}[G] J_{\rho}^{a} \partial^{\rho} \mathcal{A}_{a}+K^{a} \square_{G} \mathcal{A}_{a}-\frac{1}{2} \operatorname{Ric}[G] \nabla^{\mu} \mathcal{A}_{\mu}+\frac{1}{4} g_{Y M} e^{-\sigma / 2} \nabla_{\mu} \nabla_{\nu} \tilde{T}^{\eta \nu} \\
& =\operatorname{Ric}[G] K^{a} \mathcal{A}_{a}-\frac{3}{2} \operatorname{Ric}[G] \nabla^{\mu} \mathcal{A}_{\mu}+\frac{1}{4} \kappa R\left(2 R^{-2} T+\nabla_{\mu} \nabla_{\nu} \tilde{T}^{\mu \nu}\right)
\end{aligned}
$$


Together with

$$
\square_{G}\left(\nabla^{\mu} \mathcal{A}_{\mu}\right)=-\frac{1}{2} \operatorname{Ric}[\mathrm{G}] \nabla^{\mu} \mathcal{A}_{\mu}+\nabla^{\mu} \square_{G} \mathcal{A}_{\mu},
$$

it follows that the scalar field $\nabla^{\mu} \mathcal{A}_{\mu}$ satisfies the wave equation

$$
\square_{G}\left(\nabla^{\mu} \mathcal{A}_{\mu}\right)=-2 \operatorname{Ric}[G]\left(\nabla^{\mu} \mathcal{A}_{\mu}\right)+\operatorname{Ric}[G] \mathcal{A}^{\perp}+\frac{1}{4} \kappa R\left(2 R^{-2} T+\nabla_{\mu} \nabla_{\nu} \tilde{T}^{\mu \nu}\right) .
$$

Together with (5.32) we we obtain the following "almost-decoupled" wave equations

$$
\begin{aligned}
\square_{G \chi} \chi & =\frac{\kappa R}{4} \nabla_{\mu} \nabla_{\nu} \tilde{T}^{\mu \nu}, \\
\left(\square_{G}+\operatorname{Ric}[G]\right) \mathcal{A}^{\perp} & =-2 \operatorname{Ric}[G] \chi+\frac{1}{2} \kappa R^{-1} T
\end{aligned}
$$

where

$$
\chi:=\nabla^{\mu} \mathcal{A}_{\mu}-\mathcal{A}^{\perp}=J_{\mu}^{a} \partial^{\mu} \mathcal{A}_{a} .
$$

The second is a scalar wave equation for $\mathcal{A}^{\perp}$, and $\chi$ can be seen as part of its source, determined by the first equation. For distances below the "cosmological" scales, the mass term can be neglected, leading to massless wave equations with source determined by $T_{\mu \nu}$ as above.

A remark on the relation with the noncommutative gauge theory point of view is in order. The usual gauge fields $A_{\mu}$ in the gauge theory interpretation are related to our tangential perturbations as

$$
\theta^{\mu \nu} A_{\nu}=\eta^{\mu \nu} \mathcal{A}_{\nu}
$$

since $J_{\mu a}=\eta_{\mu a}$ if $x^{a}$ for $a=0,1$ are normal embedding coordinates, cf. (4.11). Thus

$$
\partial^{\mu} \mathcal{A}_{\mu} \sim \theta^{\mu \nu} \partial_{\mu} A_{\nu}=\frac{1}{2} \theta^{\mu \nu} F_{\mu \nu}
$$

up to some constant. This is gauge invariant (more precisely it transforms as a scalar field under noncommutative gauge transformations i.e. symplectomorphisms), and encodes the only physical degree of freedom in 2D gauge theory. Similarly, $\mathcal{A}^{\perp}$ can be interpreted as noncommutative scalar field in the noncommutative gauge theory. Therefore $\partial^{\mu} \mathcal{A}_{\mu}$ and $\mathcal{A}^{\perp}$ completely capture the physics of the system, which is described by (5.39) at the semi-classical (Poisson) level. It is also worth pointing out that the radial and tangential perturbations mix as observed in $[21,22]$, but we were able to disentangle them in the 2-dimensional case.

\section{Conclusion}

We studied the fuzzy version of 2-dimensional de Sitter and Anti-de Sitter space, and some of the associated physics. The quantization map is discussed in detail, and we obtained 
explicit formulae for the functions on the fuzzy hyperboloid corresponding to unitary irreducible representations of $\mathrm{SO}(2,1)$. This should provide the basis for further work on the associated non-commutative field theory on a curved space-time with Minkowski signature. Moreover, we consider a matrix model which admits fuzzy $(A) d S^{2}$ as solution, and study the resulting dynamics of the geometry. This allows to study the general ideas of emergent geometry in matrix models on a simple curved background with Minkowski signature. Although the model is modified as compared with the IKKT model by adding a cubic term, it is an interesting toy model which allows to essentially solve the resulting dynamics. We find that the transversal brane perturbations indeed couple to the energy-momentum tensor as emphasized in [21, 22], and we also find a mixing between tangential and transversal perturbations in the gauge theory point of view. The brane dynamics leads to a reasonable linearized gravity theory, related to Henneaux - Teitelboim gravity in 2 dimensions. It is remarkable that this happens through the bare matrix model action, without adding any gravity terms and without invoking any quantum effects. The mechanism does not require a strong-coupling regime. Even though the present toy model is not of direct physical relevance, it is nevertheless useful to clarify the dynamics of the branes and their geometry, as a step towards higher-dimensional more physical matrix models such as the IKKT model.

It would also be interesting to study a finite-dimensional realization of the matrix model numerically, following [38]. This might serve as a toy model and testing ground for the case of Minkowski signature, as a step towards the higher-dimensional case.

\section{Acknowledgments}

The work of H.S. is supported by the Austrian Fonds für Wissenschaft und Forschung under grant P24713, and the work of D.J. was supported by the PostDoc program of the Croatian Science Fundation. This collaboration was also supported by the Austrian-Croatian WTZ project HR 22/2012 of the OEAD.

\section{A Appendix: derivation of the linearized gravity equations}

We note the following identities

$$
J_{\mu}^{a} J_{a \nu}=g_{\mu \nu}, \quad J_{\mu}^{a} K_{a \nu \eta}=0
$$

as well as

$$
\begin{aligned}
\nabla^{\mu} J_{\mu}^{a} & =K^{a}, \\
\square_{G} J_{\mu}^{a} & =\partial_{\mu} \square_{G} x^{a}+\operatorname{Ric}_{\mu \nu} J^{a \nu}=-\frac{1}{2} \operatorname{Ric}[G] J_{\mu}^{a}
\end{aligned}
$$


which follows from (5.7). Then

$$
\begin{aligned}
g_{Y M}^{-2} \theta^{\mu \mu^{\prime}} \theta^{\nu \nu^{\prime}} \nabla_{\mu} \nabla_{\nu}\left(J_{\nu^{\prime}}^{a} \partial_{\mu^{\prime}} \mathcal{A}_{a}\right) & =g_{Y M}^{-2} \theta^{\mu \mu^{\prime}} \theta^{\nu \nu^{\prime}} \nabla_{\mu}\left(K_{\nu \nu^{\prime}}^{a} \partial_{\mu^{\prime}} \mathcal{A}_{a}+J_{\nu^{\prime}}^{a} \nabla_{\nu} \partial_{\mu^{\prime}} \mathcal{A}_{a}\right) \\
& =g_{Y M}^{-2} \theta^{\mu \mu^{\prime}} \theta^{\nu \nu^{\prime}}\left(K_{\mu \nu^{\prime}}^{a} \nabla_{\nu} \partial_{\mu^{\prime}} \mathcal{A}_{a}+J_{\nu^{\prime}}^{a} \nabla_{\mu} \partial_{\mu^{\prime}} \nabla_{\nu} \mathcal{A}_{a}\right) \\
& =\frac{e^{\sigma}}{2} K^{a} \square_{G} \mathcal{A}_{a}+\frac{1}{2} g_{Y M}^{-2} \theta^{\mu \mu^{\prime}} \theta^{\nu \nu^{\prime}} J_{\nu^{\prime}}^{a} \mathrm{R}_{\mu \mu^{\prime} ; \nu} \partial_{\rho} \mathcal{A}_{a} \\
& =\frac{e^{\sigma}}{2} K^{a} \square_{G} \mathcal{A}_{a}-\frac{1}{2} g_{Y M}^{-2} R^{-2} \theta^{\mu \mu^{\prime}} \theta^{\nu \nu^{\prime}} J_{\nu^{\prime}}^{a}\left(g_{\mu \nu} \delta_{\mu^{\prime}}^{\rho}-g_{\mu^{\prime} \nu} \delta_{\mu}^{\rho}\right) \partial_{\rho} \mathcal{A}_{a} \\
& =\frac{e^{\sigma}}{2} K^{a} \square_{G} \mathcal{A}_{a}+R^{-2} J_{\nu}^{a} \partial^{\nu} \mathcal{A}_{a}
\end{aligned}
$$

using (5.6), and noting that $\nabla[g]=\nabla[G]$ here. Similarly, we obtain using (5.1)

$$
\begin{aligned}
g_{Y M}^{-2} \theta^{\mu \mu^{\prime}} \theta^{\nu \nu^{\prime}} \nabla_{\mu} \nabla_{\nu}\left(J_{\mu^{\prime}}^{a} \partial_{\nu^{\prime}} \mathcal{A}_{a}\right) & =g_{Y M}^{-2} \theta^{\mu \mu^{\prime}} \theta^{\nu \nu^{\prime}} \nabla_{\mu}\left(K_{\nu \mu^{\prime}}^{a} \partial_{\nu^{\prime}} \mathcal{A}_{a}+J_{\mu^{\prime}}^{a} \nabla_{\nu} \partial_{\nu^{\prime}} \mathcal{A}_{a}\right) \\
& =\frac{e^{\sigma}}{2}\left(K^{a} \square_{G} \mathcal{A}_{a}+\partial_{\mu} K^{a} \partial^{\mu} \mathcal{A}_{a}\right) \\
& =\frac{e^{\sigma}}{2} K^{a} \square_{G} \mathcal{A}_{a}+R^{-2} J_{\nu}^{a} \partial^{\nu} \mathcal{A}_{a}
\end{aligned}
$$

Therefore

$$
e^{-\sigma} \nabla_{\mu} \nabla_{\nu} \delta_{\mathcal{A}} G^{\mu \nu}=-K^{a} \square_{G} \mathcal{A}_{a}+\operatorname{Ric}[G] J_{\nu}^{a} \partial^{\nu} \mathcal{A}_{a}
$$

Finally, we have

$$
\frac{1}{2} e^{-\sigma} G_{\mu \nu} \delta G^{\mu \nu}=e^{-\sigma} g^{\mu \nu} J_{\mu}^{a} \partial_{\nu} \mathcal{A}_{a}=J_{\mu}^{a} \partial^{\mu} \mathcal{A}_{a}
$$

Therefore

$$
\begin{aligned}
e^{-\sigma} \delta_{\mathcal{A}} \operatorname{Ric}[G] & =e^{-\sigma}\left(\square_{G}+\frac{1}{2} \operatorname{Ric}[G]\right)\left(G_{\mu \nu} \delta G^{\mu \nu}\right)-e^{-\sigma} \nabla_{\mu} \nabla_{\nu} \delta G^{\mu \nu} \\
& =\left(2 \square_{G}+\operatorname{Ric}[G]\right)\left(J_{\mu}^{a} \partial^{\mu} \mathcal{A}_{a}\right)+\left(K^{a} \square_{G} \mathcal{A}_{a}-\operatorname{Ric}[G] J_{\nu}^{a} \partial^{\nu} \mathcal{A}_{a}\right) \\
& =K^{a} \square_{G} \mathcal{A}_{a}+2 \nabla^{\mu}\left(J_{\mu}^{a} \square_{G} \mathcal{A}_{a}\right),
\end{aligned}
$$

where we used

$$
\begin{aligned}
\square_{G}\left(J_{\mu}^{a} \partial^{\mu} \mathcal{A}_{a}\right) & =\square_{G} J_{\mu}^{a} \partial^{\mu} \mathcal{A}_{a}+J_{\mu}^{a} \square_{G} \partial^{\mu} \mathcal{A}_{a}+2 K_{\mu \nu}^{a} \nabla^{\mu} \nabla^{\nu} \mathcal{A}_{a} \\
& =-\frac{1}{2} \operatorname{Ric}[G] J_{\mu}^{a} \partial^{\mu} \mathcal{A}_{a}+J_{\mu}^{a} \partial^{\mu} \square_{G} \mathcal{A}_{a}+\operatorname{Ric}_{\mu \nu}[G] J_{\mu}^{a} \partial^{\nu} \mathcal{A}_{a}+K^{a} \square_{G} \mathcal{A}_{a} \\
& =\nabla^{\mu}\left(J_{\mu}^{a} \square_{G} \mathcal{A}_{a}\right)-\left(\nabla^{\mu} J_{\mu}^{a}\right) \square_{G} \mathcal{A}_{a}+K^{a} \square_{G} \mathcal{A}_{a} \\
& =\nabla^{\mu}\left(J_{\mu}^{a} \square_{G} \mathcal{A}_{a}\right)
\end{aligned}
$$

due to (A.2). Now we can use the equations of motion (5.19), which give

$$
\begin{aligned}
\nabla^{\mu}\left(J_{\mu}^{a} \square_{G} \mathcal{A}_{a}\right) & =\frac{1}{4} g_{Y M} e^{\sigma / 2} \nabla^{\mu} g_{\mu \nu} \nabla_{\rho} \tilde{T}^{\nu \rho}=\frac{1}{4} g_{Y M} e^{-\sigma / 2} \nabla_{\mu} \nabla_{\nu} \tilde{T}^{\mu \nu} \\
K^{a} \square_{G} \mathcal{A}_{a} & =-\frac{1}{8} g_{Y M} e^{3 \sigma / 2} K^{a} K_{a} T=\frac{1}{2} g_{Y M} e^{-\sigma / 2} R^{-2} T
\end{aligned}
$$


recalling that $J^{a} K^{a}=0$, as well as

$$
K^{a} K_{a}=-4 e^{-2 \sigma} R^{-2}
$$

Putting these together, we finally arrive at

$$
\begin{aligned}
\delta_{\mathcal{A}} \operatorname{Ric}[G] & =\frac{1}{2} g_{Y M} e^{\sigma / 2}\left(R^{-2} T+\nabla_{\mu} \nabla_{\nu} \tilde{T}^{\mu \nu}\right) \\
& =8 \pi G_{N}\left(T+R^{2} \nabla_{\mu} \nabla_{\nu} \tilde{T}^{\mu \nu}\right) \\
8 \pi G_{N} & =e^{\sigma / 2} \frac{g_{Y M}}{2 R^{2}}=\frac{\kappa e^{\sigma}}{2 R} .
\end{aligned}
$$

Open Access. This article is distributed under the terms of the Creative Commons Attribution License (CC-BY 4.0), which permits any use, distribution and reproduction in any medium, provided the original author(s) and source are credited.

\section{References}

[1] J. Madore, The Fuzzy sphere, Class. Quant. Grav. 9 (1992) 69 [InSPIRE].

[2] H. Grosse, C. Klimčík and P. Prešnajder, Towards finite quantum field theory in noncommutative geometry, Int. J. Theor. Phys. 35 (1996) 231 [hep-th/9505175] [INSPIRE].

[3] H. Grosse, C. Klimčík and P. Prešnajder, Field theory on a supersymmetric lattice, Commun. Math. Phys. 185 (1997) 155 [hep-th/9507074] [INSPIRE].

[4] C.-S. Chu, J. Madore and H. Steinacker, Scaling limits of the fuzzy sphere at one loop, JHEP 08 (2001) 038 [hep-th/0106205] [INSPIRE].

[5] D. Karabali, V. Nair and A. Polychronakos, Spectrum of Schrödinger field in a noncommutative magnetic monopole, Nucl. Phys. B 627 (2002) 565 [hep-th/0111249] [INSPIRE].

[6] S. Vaidya, Scalar multi solitons on the fuzzy sphere, JHEP 01 (2002) 011 [hep-th/0109102] [INSPIRE].

[7] H. Steinacker, Quantized gauge theory on the fuzzy sphere as random matrix model, Nucl. Phys. B 679 (2004) 66 [hep-th/0307075] [INSPIRE].

[8] P. Castro-Villarreal, R. Delgadillo-Blando and B. Ydri, A Gauge-invariant UV-IR mixing and the corresponding phase transition for U(1) fields on the fuzzy sphere, Nucl. Phys. B 704 (2005) 111 [hep-th/0405201] [INSPIRE].

[9] A. Balachandran, S. Kurkcuoglu and S. Vaidya, Lectures on fuzzy and fuzzy SUSY physics, hep-th/0511114 [INSPIRE].

[10] R. Delgadillo-Blando, D. O'Connor and B. Ydri, Matrix Models, Gauge Theory and Emergent Geometry, JHEP 05 (2009) 049 [arXiv:0806.0558] [INSPIRE].

[11] P.-M. Ho and M. Li, Large-N expansion from fuzzy AdS $S_{2}$, Nucl. Phys. B 590 (2000) 198 [hep-th/0005268] [INSPIRE].

[12] P.-M. Ho and M. Li, Fuzzy spheres in AdS/CFT correspondence and holography from noncommutativity, Nucl. Phys. B 596 (2001) 259 [hep-th/0004072] [INSPIRE]. 
[13] H. Miglbauer, Kovariante nichtkommutative Näherungen nichtkompakter Geometrien und Nicht-Euklidische 2D-Fuzzy-Quantenfelder, PhD thesis, Technical University Graz, 1994, unpublished.

[14] H. Grosse, P. Prešnajder and Z. Wang, Quantum Field Theory on quantized Bergman domain, J. Math. Phys. 53 (2012) 013508 [arXiv:1005.5723] [INSPIRE].

[15] K. Hasebe, Non-Compact Hopf Maps and Fuzzy Ultra-Hyperboloids, Nucl. Phys. B 865 (2012) 148 [arXiv:1207.1968] [INSPIRE].

[16] J.-P. Gazeau and F. Toppan, A Natural fuzzyness of de Sitter space-time, Class. Quant. Grav. 27 (2010) 025004 [arXiv: 0907.0021] [INSPIRE].

[17] E. Joung, J. Mourad and R. Parentani, Group theoretical approach to quantum fields in de Sitter space. I. The Principle series, JHEP 08 (2006) 082 [hep-th/0606119] [INSPIRE].

[18] E. Joung, J. Mourad and R. Parentani, Group theoretical approach to quantum fields in de Sitter space. II. The complementary and discrete series, JHEP 09 (2007) 030 [arXiv: 0707.2907] [INSPIRE].

[19] H. Steinacker, Emergent Gravity and Noncommutative Branes from Yang-Mills Matrix Models, Nucl. Phys. B $\mathbf{8 1 0}$ (2009) 1 [arXiv:0806.2032] [InSPIRE].

[20] H. Steinacker, Emergent Geometry and Gravity from Matrix Models: an Introduction, Class. Quant. Grav. 27 (2010) 133001 [arXiv: 1003.4134] [INSPIRE].

[21] H. Steinacker, Gravity and compactified branes in matrix models, JHEP 07 (2012) 156 [arXiv: 1202.6306] [INSPIRE].

[22] H. Steinacker, The curvature of branes, currents and gravity in matrix models, JHEP 01 (2013) 112 [arXiv:1210.8364] [INSPIRE].

[23] H. Steinacker, On the Newtonian limit of emergent NC gravity and long-distance corrections, JHEP 12 (2009) 024 [arXiv:0909.4621] [INSPIRE].

[24] J.D. Brown, M. Henneaux and C. Teitelboim, Black Holes in Two Space-time Dimensions, Phys. Rev. D 33 (1986) 319 [inSPIRE].

[25] R.B. Mann, A. Shiekh and L. Tarasov, Classical and Quantum Properties of Two-dimensional Black Holes, Nucl. Phys. B 341 (1990) 134 [INSPIRE].

[26] V. Bargmann, Irreducible unitary representations of the Lorentz group, Annals Math. 48 (1947) 568 [INSPIRE].

[27] J. Repka, Tensor products of unitary representations of $S L_{2}(R)$, Bull. Amer. Math. Soc. 82 (1976) 930.

[28] A. van Tonder, Cohomology and decomposition of tensor product representations of $\mathrm{SL}(2, \mathbb{R})$, Nucl. Phys. B 677 (2004) 614 [hep-th/0212149] [INSPIRE].

[29] V.F. Molčanov, Tensor products of unitary representations of the three-dimensional Lorentz group, Math. USSR Izv. 15 (1980) 113.

[30] R.P. Martin, Tensor products for SL(2, k), Trans. Amer. Math. Soc. 239 (1978) 197.

[31] W.J. Holman and Lawrence C. Biedenharn, Complex angular momenta and the groups $\mathrm{SU}(1,1)$ and $\mathrm{SU}(2)$, Ann. Phys. 39 (1966) 1.

[32] W.J. Holman and Lawrence C. Biedenharn, A general study of the Wigner coefficients of SU(1,1), Ann. Phys. 47 (1968) 205. 
[33] M. Bordemann, E. Meinrenken and M. Schlichenmaier, Toeplitz quantization of Kähler manifolds and $g l(N), N \rightarrow \infty$ limits, Commun. Math. Phys. 165 (1994) 281 [hep-th/9309134] [INSPIRE].

[34] S.T. Ali and M. Englis, Quantization methods: A Guide for physicists and analysts, Rev. Math. Phys. 17 (2005) 391 [math-ph/0405065] [INSPIRE].

[35] D. Borthwick and A. Uribe, Almost complex structures and geometric quantization, Math. Res. Letters 3 (1996) 845 [arXiv:dg-ga/9608006].

[36] S. Waldmann, Poisson-Geometrie und Deformations-quantisierung, Springer, 2007.

[37] J. Arnlind, J. Choe and J. Hoppe, Noncommutative Minimal Surfaces, arXiv:1301.0757.

[38] S.-W. Kim, J. Nishimura and A. Tsuchiya, Expanding universe as a classical solution in the Lorentzian matrix model for nonperturbative superstring theory, Phys. Rev. D 86 (2012) 027901 [arXiv: 1110.4803] [INSPIRE]. 